\title{
Effect of Cochlear Implant Electrode Array Design on Electrophysiological and Psychophysical Measures: Lateral Wall versus Perimodiolar Types
}

\author{
Ji Young Lee ${ }^{1}$, Sung Hwa Hong ${ }^{2}$, II Joon Moon ${ }^{3,4}$, Eun Yeon Kim5, \\ Eunjoo Baek ${ }^{3}$, Hye Yoon Seol ${ }^{4,6}$, and Sihyung Kang ${ }^{2}$ \\ ${ }^{1}$ Department of Audiology and Speech-Language Pathology, Daegu Catholic University, Gyeongsan, Korea \\ ${ }^{2}$ Department of Otorhinolaryngology-Head and Neck-Surgery, Samsung Changwon Hospital, School of Medicine, \\ Sungkyunkwan University, Changwon, Korea \\ ${ }^{3}$ Department of Otorhinolaryngology-Head and Neck-Surgery, Samsung Medical Center, School of Medicine, Sungkyunkwan University, \\ Seoul, Korea \\ ${ }^{4}$ Hearing Research Laboratory of Samsung Medical Center-Samsung Electronics Co., Seoul, Korea \\ ${ }^{5}$ Department of Speech-Language Pathology, Graduate School of Social Education, Myongji University, Seoul, Korea \\ ${ }^{6}$ Sungkyunkwan University Samsung Advanced Institute for Health Sciences and Technology, Seoul, Korea
}

\begin{abstract}
Received May 7,2019
Revised May 31, 2019

Accepted June 4, 2019
\end{abstract}

\section{Address for correspondence \\ Ji Young Lee, $\mathrm{PhD}$ \\ Department of Audiology and \\ Speech-Language Pathology, \\ Daegu Catholic University, \\ 13-13 Hayang-ro, Hayang-eup, Gyeongsan 38430, Korea \\ Tel +82-53-850-2544 \\ Fax +82-53-359-6780 \\ E-mail jyslwm@gmail.com}

A portion of data in the present study was presented as a poster at the 2013 meeting of Asia Pacific Symposium on Cochlear Implants and Related Sciences, Hyderabad, India.
Background and Objectives: The present study aims to investigate whether the cochlear implant electrode array design affects the electrophysiological and psychophysical measures. Subjects and Methods: Eighty five ears were used as data in this retrospective study. They were divided into two groups by the electrode array design: lateral wall type $(\mathrm{LW})$ and perimodiolar type (PM). The electrode site was divided into three regions (basal, medial, apical). The evoked compound action potential (ECAP) threshold, T level, C level, dynamic range (DR), and aided air conduction threshold were measured. Results: The ECAP threshold was lower for the PM than for the LW, and decreased as the electrode site was closer to the apical region. The T level was lower for the PM than for the LW, and was lower on the apical region than on the other regions. The $\mathrm{C}$ level on the basal region was lower for the PM than for the LW whereas the $\mathrm{C}$ level was lower on the apical region than on the other regions. The DRs on the apical region was greater for the PM than for the LW whereas the DR was narrower on the apical region than on the other regions. The aided air conduction threshold was not different for the electrode design and frequency. Conclusions: The current study would support the advantages of the PM over the LW in that the PM had the lower current level and greater DR, which could result in more localized neural stimulation and reduced power consumption.

J Audiol Otol 2019;23(3):145-152

KEY WORDS: Cochlear implant electrode arrays · Lateral wall · Perimodiolar · ECAP . Mapping.

\section{Introduction}

Cochlear implant (CI) is an electronic medical device to aid hearing when the cochlea does not function normally due to its underdevelopment or destruction. Differently from the

This is an Open Access article distributed under the terms of the Creative Commons Attribution Non-Commercial License (https:/creativecommons.org/licenses/by-nc/4.0/) which permits unrestricted non-commercial use, distribution, and reproduction in any medium, provided the original work is properly cited. normal auditory system where the sound is transmitted through the sensory hair cells to the auditory nerve, CI gets sound information directly to the auditory nerve by exciting neurons with electrical stimuli, bypassing the damaged hair cells in the cochlea [1]. CI has been a good option when conventional hearing aids cannot work enough to deal with the hearing loss. In particular, since a multichannel CI was developed in industry in 1982, people with multichannel CI system have shown significant performance on discriminating speech sounds 
without visual cues and developing language skills [2].

The perceptual outcome of CI can be influenced by a variety of factors encompassing anatomical, audiological, surgical, and technical aspects. There are still some issues to discuss with these factors, one of which is electrode array design. The electrode is a crucial factor in that it contacts directly with the introcochlear tissues that line neural elements. The placement of the electrode array within the cochlea can potentially affects the activation of the auditory nerve $[3,4]$.

With surgical and technical advances of CI, new designs of lateral wall type (LW) and perimodiolar type (PM) of electrode arrays have been devised to effectively and efficiently stimulate the auditory nerve. For over three decades, the lateral wall or straight type of electrode array intended to lie along the lateral wall of the scala tympani has been developed by all CI manufacturers. The LW is believed to stimulate the neural fiber endings of the auditory neurons at the Organ of Corti [5]. The LW has been used for the population with a variety of anatomical variations when the structure of the cochlea does not suit for a perimodiolar electrode design. More recently, the thinner and flexible LWs were designed to be inserted with minimal trauma. The LW has also shown useful preservation of residual hearing, facilitating benefits from combined electric and acoustic stimulation [6,7].

The PM of electrode array, on the other hand, is believed to stimulate the spiral ganglion cells by positioning the electrode closer to the modiolus. The PM, so called modiolus-hugging or contour type, has shown some advantages over the LW in lower stimulation levels, expanded dynamic rage, and better channel separation. It seems to lead to better localized neural stimulation because the voltage could be decreased, which might result in better speech perception [8]. The PM, however, is more likely to cause trauma during insertion due to its greater volume and stiffness [5] and its pre-curved design may not match the coiling pattern of individual cochlear morphologies [2].

Modeling and animal studies suggested that it is more beneficial for the electrode array to be positioned near the spiral ganglion in the modiolus because it results in the lowered threshold, wider dynamic range (DR), and reduced channel interaction. Some computational modeling research have reported that when the electrode was placed distant from the neurons or near degenerative regions of the neurons, the higher current level (CL) was required and the broader region was excited, which could increase channel interaction and distortion of spectral information [9,10]. Shepherd, et al. [11] recorded the electrically evoked auditory brainstem response (EABR), varying the position of the electrode array within the scala tympani in ten cats. Their results showed that the EABR threshold became significantly reduced as the electrode array was moved from the lateral wall towards the modiolus, and it was further reduced when the electrode was placed underneath the osseous spiral lamina. Because of the difficulties to achieve the site of osseous spiral lamina in practice, they concluded the electrode placement adjacent to the modiolus would result in more localized neural excitation and increased DR, which may improve speech perception.

Clinical studies in human CI users have been also conducted on electrophysiological and behavior measurements. The electrically evoked compound action potential (ECAP) has been widely used as a valuable objective tool in mapping the CI, especially for those who have difficulty in showing the reliable behavioral response [12]. Whereas some studies reported that the ECAP threshold, T level, and C level were lower for the PM than the LW, and were lower on the apical region than on the basal and medial regions [13-15], other studies showed different results $[16,17]$. These contrasting results may be because only a small number of subjects or subjects with much heterogeneity participated, only several electrode contacts were selected to represent the electrode site, and more recent LWs were included or exclude in the studies. In particular, it would be interesting to include the more recent thinner and flexible LW because it was intended to cause less trauma, which may stimulate more surviving neural elements whereas it is still positioned toward the lateral wall of the cochlea, which may limit the access to the nerve fibers.

The current clinical study was conducted in attempt to minimize the limitations of the previous studies by including more subjects with less heterogeneity, all of 22 active electrode contacts, and CI422 for the LW. The present study aims to investigate the effect of CI electrode array design on the electrophysiological and psychophysical measures by determining whether the electrode design and electrode site affect the ECAP threshold, T level, C level, and DR, and whether the electrode design and frequency affect the aided air conduction threshold.

\section{Subjects and Methods}

\section{Subjects}

Eighty CI users who were implanted at the Samsung Medical Center between 2010 and 2013 and met the criteria below participated in this retrospective study. The subjects had severe to profound hearing loss in the ear implanted before. They all used Nucleus 5 sound processor (Cochlear Ltd., Lane Cove, Australia) and advanced combination encoder (ACE) strategy, and had implant experience for at least 6 months. All of 22 electrodes were fully inserted and were active dur- 
ing the psychophysical measurement at 6 months after surgery. People whose ages were older than 59 years old or had missing data for the electrophysiological and psychophysical measures needed for the present study were excluded. The type of electrode array was chosen by the surgeon's professional judgement based on the residual hearing and anatomical configuration. Included were five subjects bilaterally implanted and tested on both ears. Thus, 85 ears were analyzed in the present study, being divided into two groups by the electrode design: LW and PM. LW group was implanted with the Nucleus Slim Straight (CI422, n=31) (Cochlear Ltd.), and PM group was implanted with Nucleus Contour Advance (CI512, n=44) (Cochlear Ltd.) or Nucleus Freedom Contour Advance (CI24RE, n=10) (Cochlear Ltd.). When the chi square test was administered, there were no significant differences for the ratios of the onset of deafness and gender between LW and PM, respectively $\left[\chi^{2}(1)=1.692, p=0.193\right.$; $\left.\chi^{2}(1)=1.219, p=0.270\right]$. Also, the independent t-test showed that there was no significant difference for the age between LW and PM $[t(83)=0.494, p=0.624]$. Demographic information is seen in Table 1.

\section{Procedure}

To examine the effects of the electrode design and electrode site, the electrophysiological and psychophysical responses were measured. The present study was approved by the Samsung Medical Center Institutional Review Board (IRB No. 2019-02-087).

For the electrophysiological measures, the ECAP threshold was obtained at electrode 1, 3, 5, 7, 9, 11, 13, 15, 17, 19, and 21 via neural response telemetry (NRT) intraoperatively at the day of CI surgery. The ECAP threshold was measured as the lowest CL to evoke measurable ECAP, using AutoNRT. The CL, the amount of electrical current delivered to the CI users, is the clinical programing unit to measure the ECAP threshold. For the statistical analysis, a threshold value of $255 \mathrm{CL}$, the maximum stimulating CL, was assigned to the cases where the ECAP was absent during the test.

For the psychophysical measures, the T level, C level, and aided air conduction threshold were obtained with all of 22 active electrodes during the regular clinical session at 6 months follow up after CI surgery. The T level was measured as the minimum amount of electrical CL needed for each electrode so that the CI user can detect as the softest sound, and the Clevel was measured as the maximum amount of electrical CL needed for each electrode so that he or she can tolerate as the comfortable sound. The DR, the current intensity span between the $\mathrm{T}$ level and $\mathrm{C}$ level, was calculated by subtracting the value of $\mathrm{T}$ level from the value of $\mathrm{C}$ level. The aided air conduction threshold was obtained, presenting warble tones through loudspeaker positioned at $1 \mathrm{~m}$ from the subject in a soundproof booth.

\section{Statistical analysis}

IBM SPSS Statistics 19 (IBM Corp., Armonk, NY, USA) was used for the statistical analysis. In conducting analysis of variances (ANOVAs), Greenhouse-Geisser adjusted degrees of freedom, F- and $p$-values were reported when Mauchly's test of sphericity was significant. Bonferonni corrections were used for any subsequent univariate testing. An alpha level of 0.05 was considered significant.

\section{Electrophysiological measures}

A two way mixed ANOVA was administered on the ECAP threshold with the electrode design (2 levels: LW, PM) and electrode site (3 levels: basal, medial, apical) as the factors. To analyze the general tendency of the electrode site, the electrode site was divided into the basal (electrode 1 to 7), medial (electrode 9 to 15), and apical regions (electrode17 to 21) on electrode $1,3,5,7,9,11,13,15,17,19$, and 21 where the ECAP threshold was obtained. The ECAP threshold on each region was obtained by averaging the values of the ECAP threshold at the corresponding electrode contacts. For example, the ECAP threshold on the basal region was calculated by averaging the ECAP thresholds at electrode 1, 3, 5, and 7 .

\section{Psychophysical measures}

The two-way mixed ANOVAs were conducted on the $\mathrm{T}$ level, C level, and DR, respectively, with the electrode design (2 levels: LW, PM) and electrode site (3 levels: basal, medial, apical) as the factors. The electrode site was divided into the basal (electrode 1 to 8), medial (electrode 9 to 15), and apical regions (electrode 16 to 22 ) on electrode 1 to 22 where the $\mathrm{T}$ level, C level, and DR were obtained. The T level, C level,

Table 1. Demographic information

\begin{tabular}{|c|c|c|c|}
\hline & Onset of deafness (Prelingual:Postlingual) (n) & Gender (Male:Female) (n) & $\begin{array}{c}\text { Age at implantation (years) } \\
\text { [mean (range)] }\end{array}$ \\
\hline $\operatorname{LW}(n=31)$ & $25: 6$ & $10: 21$ & $10.1(1.1-56.5)$ \\
\hline$P M(n=54)$ & $49: 5$ & $24: 30$ & $7.0(1.0-53.9)$ \\
\hline$p$-value & 0.193 & 0.270 & 0.624 \\
\hline
\end{tabular}

LW: lateral wall type, PM: perimodiolar type 
and DR on each region were obtained by averaging their values at the corresponding electrode contacts, respectively. For example, the $\mathrm{T}$ level on the basal region was calculated by averaging the T levels at electrode $1,2,3,4,5,6,7$, and 8 .

With the exclusion of 6 missing data ( 5 in LW group, 1 in PM group), a two-way mixed ANOVA was used on the aided air conduction threshold with the electrode design (2 levels: LW, PM) and frequency (3 levels: $500 \mathrm{~Hz}, 1,000 \mathrm{~Hz}, 2,000$ $\mathrm{Hz})$ as the factors.

\section{Results}

\section{ECAP threshold}

The ECAP threshold was significantly different for both the electrode design $\left(F_{1,83}=4.105, p=0.046, \eta p^{2}=0.047\right)$ and electrode site $\left(F_{1.823,151.328}=13.630, p<0.001, \eta p^{2}=0.141\right)$ with no interaction effect between them. For the electrode design, the ECAP threshold was lower for PM than for LW. For the electrode site, the ECAP threshold was lower on the apical region versus the basal and medial regions, and was not different between the basal and medial regions. Fig. 1 shows that the ECAP threshold for the electrode design and electrode site. Fig. 2 shows that the ECAP threshold at individual electrode contacts by the electrode design in more details.
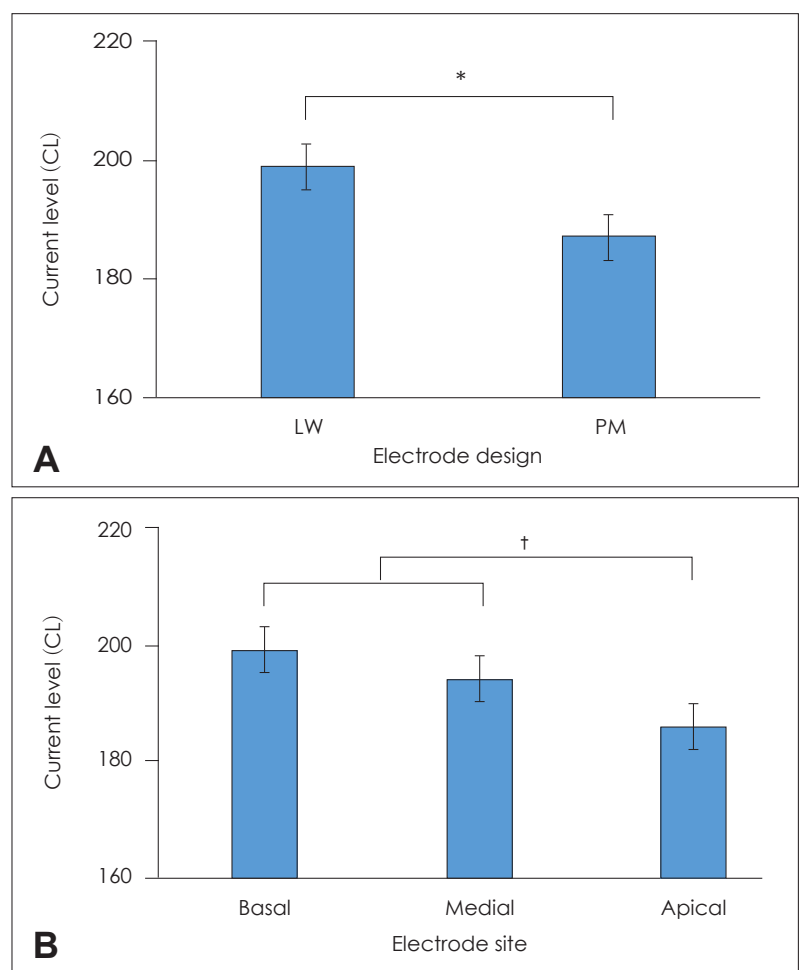

Fig. 1. Evoked compound action potential threshold for $(A)$ electrode design and $(B)$ electrode site. Error bars indicate 1 standard error from the mean. ${ }^{*} p<0.05,{ }^{\dagger} p<0.001$. CL: current level, LW: lateral wall type, PM: perimodiolar type.

\section{T level, C level, and DR}

The $\mathrm{T}$ level was significantly different for both the electrode design $\left(F_{1,83}=13.989, p<0.001, \eta p^{2}=0.144\right)$ and electrode site $\left(F_{1.491,123.738}=5.889, p=0.008, \eta p^{2}=0.066\right)$ with no interaction effect between them. For the electrode design, the T level was lower for PM than for LW. For the electrode site, the $\mathrm{T}$ level was lower on the apical region than the basal and medial regions, and was not different between the basal and medial regions. Fig. 3 shows the T level for the electrode design and electrode site.

For the $\mathrm{C}$ level, the effects of both the electrode design $\left(F_{1,83}=\right.$ 5.046, $\left.p=0.270, \eta p^{2}=0.057\right)$ and electrode site $\left(F_{1.326,110.054}=\right.$ $\left.102.768, p<0.001, \eta p^{2}=0.563\right)$ were significant, and the interaction between them was also significant $\left(F_{1.326,110.054}=6.341\right.$,

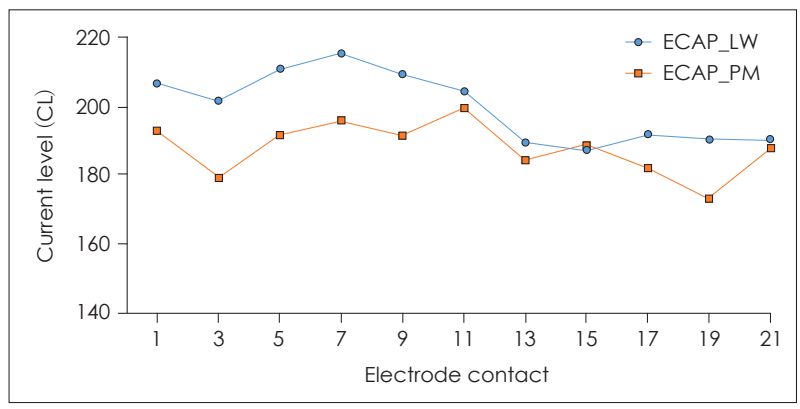

Fig. 2. Evoked compound action potential (ECAP) threshold at individual electrode contacts by electrode design. CL: current level, LW: lateral wall type, PM: perimodiolar type.

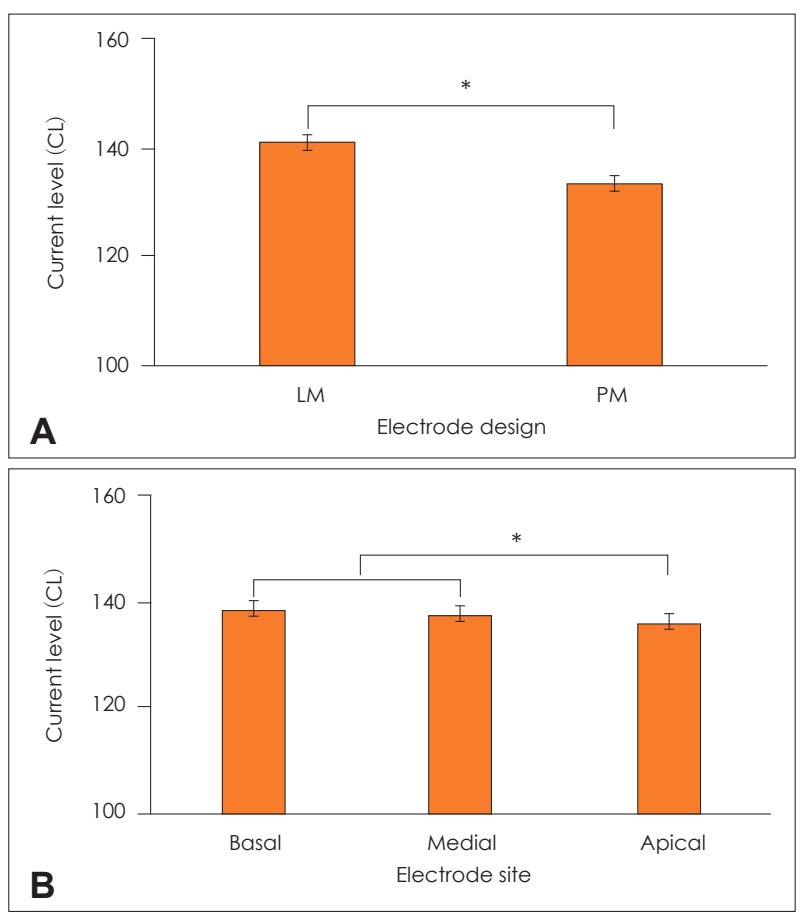

Fig. 3. T level for (A) electrode design and (B) electrode site. Error bars indicate 1 standard error from the mean. ${ }^{*} p<0.05$. CL: current level, LW: lateral wall type, PM: perimodiolar type. 
$\left.p=0.008, \eta p^{2}=0.071\right)$. The results of post-hoc tests of interaction effect are as follows. For the electrode design, the $\mathrm{C}$ level on the basal region was significantly lower for PM than for LW $[t(44.24)=2.434, p=0.019]$ while the $\mathrm{C}$ levels on the apical and medial regions were not different between LW and PM. For the electrode site, on the other hand, the C level was significantly lower on the apical region than on the basal and medial regions $\left(F_{1.425,42.743}=50.604, p<0.001, \eta p^{2}=0.628 ; F_{1.233,65.365}=\right.$ 48.279, $p<0.001, \eta p^{2}=0.477$ ), and was not different between the basal and medial regions. Fig. 4 shows the $\mathrm{C}$ level for the electrode design and electrode site. The $\mathrm{T}$ and $\mathrm{C}$ levels at individual electrode contacts by the electrode design are seen in Fig. 5 in more details.

The DR was significantly different for the electrode site $\left(F_{1.752,145.423}=39.856, p<0.001, \eta p^{2}=0.324\right)$, and showed significant interaction effect between the electrode design and electrode site $\left(F_{1.752,145.423}=8.096, p=0.001, \eta p^{2}=0.089\right)$. The results of post-hoc tests of interaction effect are as follows. For the electrode design, the DR on the apical region was wider for PM than for LW $[t(83)=2.372, p=0.020]$ while the DRs on the basal and medial regions was not different between

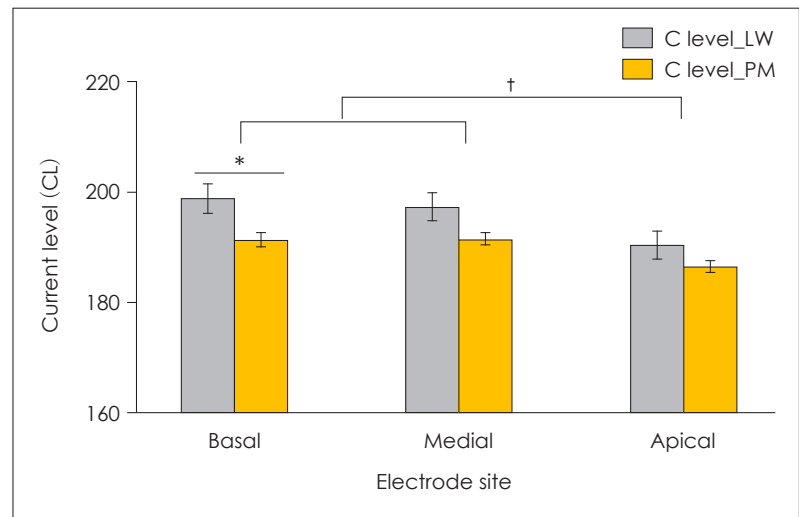

Fig. 4. C level for electrode design and electrode site. Error bars indicate 1 standard error from the mean. ${ }^{*} p<0.05,{ }^{\dagger} p<0.001$. CL: current level, LW: lateral wall type, PM: perimodiolar type.

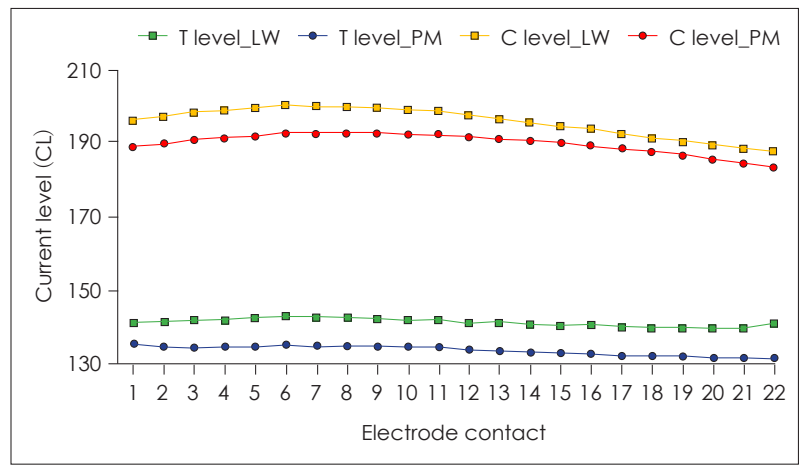

Fig. 5. $\mathrm{T}$ and $\mathrm{C}$ levels at individual electrode contacts by electrode design. CL: current level, LW: lateral wall type, PM: perimodiolar type.
LW and PM. For the electrode site, on the other hand, the DR was significantly narrower on the apical region than on the basal and medial regions $\left(F_{2,60}=30.143, p<0.001, \eta p^{2}=0.501\right.$; $\left.F_{1.513,80.188}=10.972, p=0.001, \eta p^{2}=0.172\right)$, and was not different between the basal and medial regions. Fig. 6 shows the DR for the electrode design and electrode site. Fig. 7 shows the DR at individual electrode contacts by the electrode design in more details. Table 2 shows the mean and standard deviation (SD) of the ECAP threshold, T level, C level, and DR for the electrode design and electrode site.

\section{Aided sound field threshold}

The aided air conduction threshold was not different for the electrode design and frequency. Table 3 shows the aided air conduction threshold for the electrode design and frequency.

\section{Discussion}

The ECAP threshold was lower for PM than for LW whereas it was lower on the apical region than on the basal and medial regions. It is consistent with some previous findings [1719]. Telmesani and Said [17] showed that the ECAP threshold

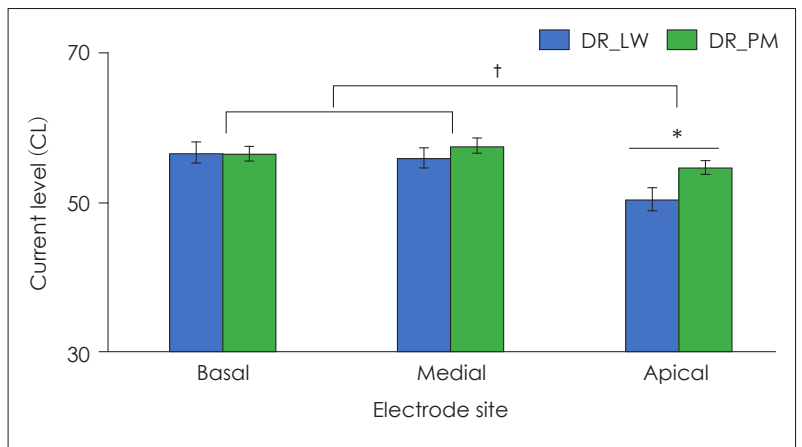

Fig. 6. DR for electrode design and electrode site. Error bars indicate 1 standard error from the mean. ${ }^{*} p<0.05,{ }^{\dagger} p<0.001$. CL: current level, DR: dynamic range, LW: lateral wall type, PM: perimodiolar type.

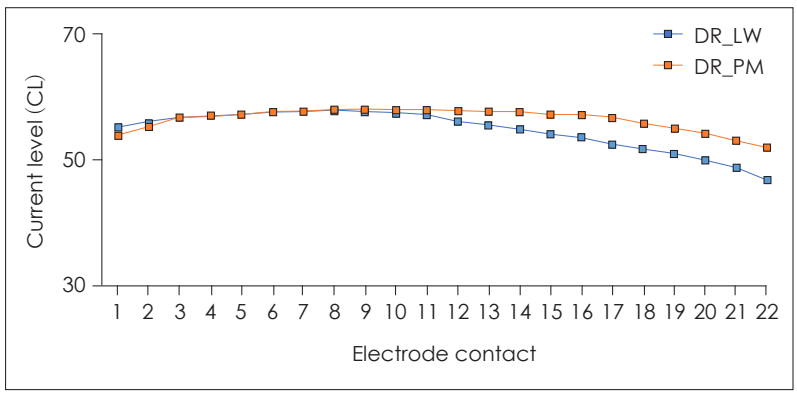

Fig. 7. DR at individual electrode contacts by electrode design. CL: current level, DR: dynamic range, LW: lateral wall type, PM: perimodiolar type. 
Table 2. ECAP threshold, T level, $C$ level, and DR (current level) for electrode design and electrode site

\begin{tabular}{cccccc}
\hline & Electrode site & ECAP threshold & Tlevel & C level & DR \\
\hline LW & Basal & $208.73(24.12)$ & $142.15(13.38)$ & $198.89(15.36)$ & $56.75(8.31)$ \\
& Medial & $197.68(27.07)$ & $141.34(12.21)$ & $197.35(14.95)$ & $56.00(7.76)$ \\
& Apical & $191.05(35.98)$ & $139.99(12.53)$ & $190.48(14.44)$ & $50.49(9.01)$ \\
PW & Basal & $189.94(27.69)$ & $134.83(7.82)$ & $191.44(9.78)$ & $56.62(7.75)$ \\
& Medial & $190.95(28.06)$ & $133.94(7.39)$ & $191.57(8.75)$ & $57.63(8.00)$ \\
& Apical & $181.07(30.58)$ & $131.96(7.68)$ & $186.63(8.71)$ & $54.67(7.07)$ \\
\hline
\end{tabular}

Values are presented as mean (standard deviation). ECAP: evoked compound action potential, DR: dynamic range, LW: Iateral wall type, PM: perimodiolar type

Table 3. Aided air conduction threshold ( $\mathrm{dB} \mathrm{HL})$ for electrode design and frequency

\begin{tabular}{cccc}
\hline & $500 \mathrm{~Hz}$ & $1,000 \mathrm{~Hz}$ & $2,000 \mathrm{~Hz}$ \\
\hline LW & $27.69(6.20)$ & $26.54(6.13)$ & $27.88(9.07)$ \\
PM & $27.74(7.31)$ & $26.98(6.23)$ & $28.30(6.86)$ \\
\hline
\end{tabular}

Values are presented as mean (standard deviation). LW: lateral wall type, PM: perimodiolar type

was lower for PM than for LW at the basal site of electrode in the pediatric CI users when the ECAP response was measured on the apical (electrode 22), mid (electrode 16 and 11), and basal regions (electrode 6 and 1). In another study, the ECAP threshold was lowest on the apical end position (electrode 22) and highest on the basal end position (electrode 1) whereas it decreased with time and remained stable at 3 months poststimulation [18]. van de Heyning [19] also reported that the ECAP amplitude was greater and the ECAP slope was steeper on the apical region than on the basal region, and the ECAP threshold was lower on the medial region than on the basal region of the cochlea.

Although it is not known exactly why such differences in the ECAP response was obtained along the cochlea, these findings may be attributed to two things. One is the proximity of electrode to the modiolus, and the other is the density and integrity of surviving spiral ganglion cells at the electrode sites $[17,20,21]$. Thus, the closer distance between the modiolus and electrode may result in the lower ECAP threshold. The measurement of X-ray showed that the perimodiolar electrode array is actually positioned closer to the modiolus than straight electrode array in the adult CI users [20]. A greater neural survival on the apical region may also lead a lower ECAP threshold compared to the other regions.

In addition, the relatively high value of the ECAP threshold was reported in the present study. It may be because the ECAP threshold was measured at the day of surgery so the neural synchrony in the primary auditory nerve has not yet improved. A greater number of neurons would be activated to elicit a response with synchrony by continuous stimulation of CI [22]. It was reported that the postoperative ECAP threshold decreased significantly, compared to the intraoperative ECAP threshold [17].

The T level was lower for PM than for LW, and was lower on the apical region than on the other regions. It is in line with other previous conclusions that the perimodiolar electrode array may result in decreased $\mathrm{T}$ and $\mathrm{C}$ levels with the lower power consumption $[11,13]$. Thus, the perimodiolar electrode arrays seems to consume the power more efficiently and stimulate spiral ganglia more specifically by the closer placement of the electrode to the modiolus. To place the electrode array as close to the modiolus would minimize the current dissemination and the spatial overlap, which would result in the reduction of the channel interaction, then possibly better frequency selectivity and speech perception $[11,13,23]$.

The $\mathrm{C}$ level on the basal region was lower for PM than for LW whereas the C level was lower on the apical region than on the other regions. It was interesting to see that the $\mathrm{C}$ level on the basal region only, not on the other regions, was lower for PM than for LW. It may be interpreted in relation to the shape of cochlea and the resulting distance change between the electrode and the modiolus along the electrode length. The diameter of the cochlea or the width of the scala tympani gets narrower toward the apex [24]. The distance between the electrode and the modioluus becomes narrower toward the apical region accordingly, and this tendency is relatively more noticeable for LW. Therefore, the electrode to modiolus distance for LW and PM may not be significantly different on the apical and medial regions whereas it is significantly different on the basal region.

The DR on the apical region was wider for PM than for LW whereas the DR was narrower on the apical region than on the other regions. As mentioned above, it may result from the relatively more decreased $\mathrm{C}$ level for $\mathrm{LW}$ on the apical region, which may make the DR gets narrower for LW than for $\mathrm{PM}$ on the apical region. It is in accordance with other previous findings. Some clinical studies have shown that the wider DR with reduced behavioral and ECAP threshold were obtained for PM, compared to LW $[15,25,26]$.

The aided air conduction threshold was not different for the electrode design. It is in agreement with Telmesani and 
Said' findings [17] that there was no significant difference on the aided air conduction threshold between LW and PM. It may be because the aided air conduction threshold is optimized by adjusting the $\mathrm{T}$ level and other mapping parameters in case it is greater than the criteria (i.e. 15 to $30 \mathrm{~dB} \mathrm{HL}$ ) [27]. The current results showed that the aided air conduction threshold was 25 to $30 \mathrm{~dB}$ HL at 500, 1,000, and 2,000 $\mathrm{Hz}$, respectively, which is consistent with the Skinner et al.'s finding [28] that the aided air conduction thresholds have been between 15 and $30 \mathrm{~dB}$ HL in CI users.

As a result, the current study would support the advantages of PM over LW on the ECAP threshold, T level, C level, and DR. The PM had the lower CL and greater DR, which could result in more localized neural stimulation and reduced power consumption. According Gibson and Boyd [6], PM is recommended for the traditional CI candidates whereas the LW, hybrid electrode is required for the people with mild to moderate low frequency hearing loss. Either LW or PM could be chosen for people with congenital anatomical abnormalities, fibrosis, ossification of the cochlea, or tumor based on their anatomical and medical conditions. The current findings may provide some useful information when there is no strong indication to select either LW or PM. In the light of some limitations of the current study, further research would need to group the subject by age, include more recent electrode arrays, consider the residual hearing and anatomical abnormalities, and analyze speech understanding as well.

\section{Conflicts of interest}

The authors have no financial conflicts of interest.

\section{Author Contributions}

Conceptualization: Sung Hwa Hong. Data curation: Eun Yeon Kim, Eunjoo Baek, and Hye Yoon Seol. Formal analysis: Sihyung Kang and Ji Young Lee. Investigation: Il Joon Moon and Ji Young Lee. Methodology: Ji Young Lee. Writing — original draft: Ji Young Lee. Writing — review \& editing: Ji Young Lee.

\section{ORCID iDs}

Ji Young Lee

Sung Hwa Hong

Il Joon Moon

Eun Yeon Kim

Eunjoo Baek

Hye Yoon Seol

Sihyung Kang https://orcid.org/0000-0001-7072-7123

https://orcid.org/0000-0001-6906-8925

https://orcid.org/0000-0002-3613-0734

https://orcid.org/0000-0002-7382-6271

https://orcid.org/0000-0002-6198-6200

https://orcid.org/0000-0002-7040-1884

https://orcid.org/0000-0002-6777-839X

\section{REFERENCES}

1) Paul PV, Whitelaw GM. Hearing and deafness: an introduction for health and education professionals. Sudbury, USA: Jones and Bartlett Publishers;2011. p109-38.

2) Rebscher SJ, Hetherington A, Bonham B, Wardrop P, Whinney D, Leake PA. Considerations for design of future cochlear implant elec- trode arrays: electrode array stiffness, size, and depth of insertion. J Rehabil Res Dev 2008;45:731-47.

3) Bierer JA. Probing the electrode-neuron interface with focused cochlear implant stimulation. Trends Amplif 2010;14:84-95.

4) Wanna GB, Noble JH, Carlson ML, Gifford RH, Dietrich MS, Haynes DS, et al. Impact of electrode design and surgical approach on scalar location and cochlear implant outcomes. Laryngoscope 2014;124 Suppl 6:S1-7.

5) Skarzynski H, Lorens A, Matusiak M, Porowski M, Skarzynski $\mathrm{PH}$, James CJ. Partial deafness treatment with the nucleus straight research array cochlear implant. Audiol Neurootol 2012;17:82-91.

6) Gibson P, Boyd P. Optimal electrode design: straight versus perimodiolar. Eur Ann Otorhinolaryngol Head Neck Dis 2016;133 Suppl 1:S63-5.

7) Sennaroglu L. Cochlear implantation in inner ear malformations--a review article. Cochlear Implants Int 2010;11:4-41.

8) Gstoettner WK, Adunka O, Franz P, Hamzavi J Jr, Plenk H Jr, Susani M, et al. Perimodiolar electrodes in cochlear implant surgery. Acta Otolaryngol 2001;121:216-9.

9) Goldwyn JH, Bierer SM, Bierer JA. Modeling the electrode-neuron interface of cochlear implants: effects of neural survival, electrode placement, and the partial tripolar configuration. Hear Res 2010;268: 93-104.

10) Hughes ML. A re-evaluation of the relation between physiological channel interaction and electrode pitch ranking in cochlear implants. J Acoust Soc Am 2008;124:2711-4.

11) Shepherd RK, Hatsushika S, Clark GM. Electrical stimulation of the auditory nerve: the effect of electrode position on neural excitation. Hear Res 1993;66:108-20.

12) Raghunandhan S, Ravikumar A, Kameswaran M, Mandke K, Ranjith R. Electrophysiological correlates of behavioral comfort levels in cochlear implantees: a prospective study. Indian J Otolaryngol Head Neck Surg 2015;67:210-22.

13) Frijns JH, de Snoo SL, Schoonhoven R. Potential distributions and neural excitation patterns in a rotationally symmetric model of the electrically stimulated cochlea. Hear Res 1995;87:170-86.

14) Parkinson AJ, Arcaroli J, Staller SJ, Arndt PL, Cosgriff A, Ebinger $\mathrm{K}$. The nucleus 24 contour cochlea implant system: adult clinical trial results. Ear Hear 2002;23(1 Suppl):41S-8S.

15) Cohen LT, Saunders E, Clark GM. Psychophysics of a prototype peri-modiolar cochlear implant electrode array. Hear Res 2001;155: 63-81.

16) Hughes ML, Abbas PJ. Electrophysiologic channel interaction, electrode pitch ranking, and behavioral threshold in straight versus perimodiolar cochlear implant electrode arrays. J Acoust Soc Am 2006; 119:1538-47.

17) Telmesani LM, Said NM. Effect of cochlear implant electrode array design of auditory nerve and behavioral response in children. Int J Pediatr Otorhinolaryngol 2015;79:660-5.

18) Spivak L, Auerbach C, Vambutas A, Geshkovich S, Wexler L, Popecki B. Eletrical compound action potentials recorded with automated neural response telemetry: threshold changes as a function of time and electrode position. Ear Hear 2011;32:104-13.

19) van de Heyning P, Arauz SL, Atlas M, Baumgartner WD, Caversaccio M, Chester-Browne R, et al. Electrically evoked compound action potentials are different depending on the site of cochlear stimulation. Cochlear Implants Int 2016;17:251-62.

20) Huang TC, Reitzen SD, Marrinan MS, Waltzman SB, Roland JT. Modiolar coiling, electrical thresholds, and speech perception after cochlear implantation using the nucleus contour advance electrode with the advance off stylet technique. Otol Neurotol 2006;27:159-66.

21) Guedes MC, Weber R, Gomez MV, Neto RV, Peralta CG, Bento RF. Influence of evoked compound action potential on speech perception in cochlear implant users. Braz J Otorhinolaryngol 2007;73: 439-45.

22) Gordon KA, Papsin BC, Harrison RV. Activity-dependent develop- 
mental plasticity of the auditory brain stem in children who use cochlear implants. Ear Hear 2003;24:485-500.

23) Saunders E, Cohen L, Aschendorff A, Shapiro W, Knight M, Stecker $\mathrm{M}$, et al. Threshold, comfortable level and impedance changes as a function of electrode-modiolar distance. Ear Hear 2002;23(1 Suppl): 28S-40S.

24) Ramos de Miguel Á, Argudo AA, Borkoski Barreiro SA, Falcón González JC, Ramos Macías A. Imaging evaluation of electrode placement and effect on electrode discrimination on different cochlear implant electrode arrays. Eur Arch Otorhinolaryngol 2018;275: 1385-94.
25) van Wermeskerken GK, van Olphen AF, Graamans K. Imaging of electrode position in relation to electrode functioning after cochlear implantation. Eur Arch Otorhinolaryngol 2009;266:1527-31.

26) Gordin A, Papsin B, James A, Gordon K. Evolution of cochlear implant arrays result in changes in behavioral and physiological response in children. Otol Neurotol 2009;30:908-15.

27) Rakszawski B, Wright R, Cadieux JH, Davidson LS, Brenner C. The effects of preprocessing strategies for pediatric cochlear implant recipients. J Am Acad Audiol 2016;27:85-102.

28) Skinner MW. Optimizing cochlear implant speech performance. Ann Otol Rhinol Laryngol Suppl 2003;191:4-13. 